\title{
An Integrated LTCC Laminated Waveguide-to-Microstrip Line T-Junction
}

\author{
Yong Huang, Ke-Li Wu, Senior Member, IEEE, and Michael Ehlert
}

\begin{abstract}
In this paper, an integrated low-temperature co-fired ceramic (LTCC) laminated waveguide to microstrip line T-junction is proposed and experimentally verified. A multilayer bellshape probe end comprising multiple parallel metallic pads is used to improve the bandwidth and matching condition. The experimental result shows good correlation with the simulated result. The configuration can also be used as the transition between microstrip line and laminated waveguide.
\end{abstract}

Index Terms-Laminated waveguide, low-temperature co-fired ceramic (LTCC), microstrip line, T-junction, transition.

\section{INTRODUCTION}

$\mathbf{L}$ OW-TEMPERATURE co-fired ceramic (LTCC) technology offers an optimal means to integrating a patch antenna array with its associated mm-wave circuits on one piece of substrate [1], [2]. Although microstrip line is a simple option for feeding network, its high surface wave loss at $\mathrm{mm}$-wave band in an LTCC substrate restricts the size of an array antenna. The laminated waveguide [3], on the other hand, eliminates the surface wave loss and is a preferable solution for low loss feeding network. However, as compared to the size of patch element, laminated waveguide is still too bulky to feed each element directly. To consolidate the features of laminated waveguide and patch array antenna, a mixed feeding network that consists of laminated waveguide and microstrip line such as the one shown in Fig. 1, can be used, in which the major trunk of the feeding network is implemented by laminated waveguide, whereas the branch subnetworks are constructed by microstrip line. In addition, efficient laminated waveguide to microstrip line $\mathrm{T}$-junctions are required as the interconnection between the two kinds of transmission lines.

A few types of interconnection between laminated waveguide and microstrip line have been reported, e.g., a planar form microstrip to laminated waveguide transition [4] and a transition via a probe partially inserted into the laminated waveguide [5]. However, the former is a full planar structure, in which microstrip line is on the same layer as that of the top wall of laminated waveguide and the performance of the latter is limited by available probe depth and diameter.

In this paper, a novel integrated $\mathrm{T}$-junction structure from a laminated waveguide to a microstrip line is presented. The sim-

Manuscript received November 4, 2002; revised February 23, 2003. The review of this letter was arranged by Associate Editor Dr. Shigeo Kawasaki.

Y. Huang and K.-L. Wu are with the Department of Electronic Engineering, The Chinese University of Hong Kong, Shatin, Hong Kong, P.R.C. (e-mail: klwu@ee.cuhk.edu.hk).

M. Ehlert is with the LTCC Division, National Semiconductor Corp., Irvine, CA 92618 USA.

Digital Object Identifier 10.1109/LMWC.2003.815690

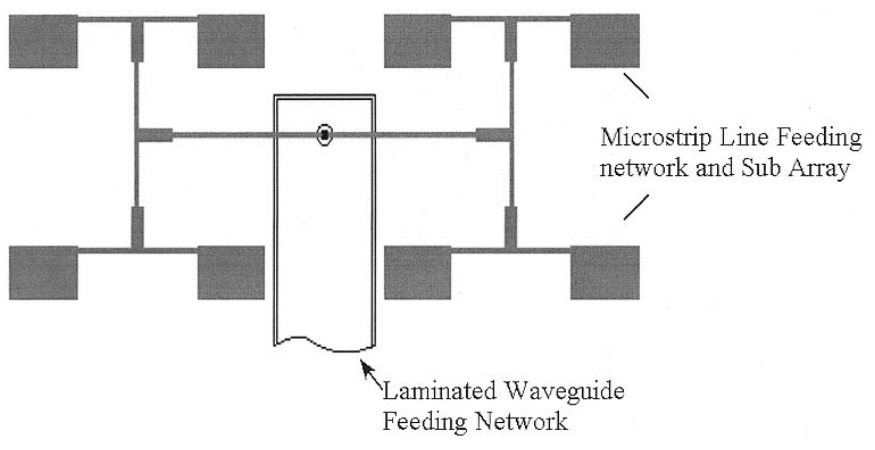

Fig. 1. Mixed feeding network of an LTCC integrated patch antenna array.

ulated results show that a more than $10 \%$ bandwidth defined at $-15 \mathrm{~dB}$ return loss can be obtained. The measured result agrees well with the simulated result.

\section{Design OF THE T-JunCtion}

The concept of the proposed T-junction is based on the traditional probe excitation for transferring energy between coaxial line and waveguide. To reduce the inductance caused by the probe penetrated in the waveguide and to provide a better impedance matching, a bell-shape probe end was suggested [6].

To use the concept, a few of metallic pads with a radius profile are stacked at the end of a probe as shown in Fig. 2. A filled via-hole is employed as the probe. As shown in Fig. $2(\mathrm{~b}), \mathrm{R}_{1}$ denotes the radius of the opening on the top conductor wall of laminated waveguide, through which the probe penetrates inside the laminated waveguide; $R_{2}$ through $R_{5}$ denotes the radius of the metallic pads for replacing the solid bell-shape probe end. The performance of the T-junction can be adjusted by the distance between the grid-like shorting wall of the laminated waveguide to the probe, radius of the probe and the geometry of bell-shape end.

A proposed T-junction working in the Local Multi-point Distribution System (LMDS) frequency band is developed with Dupont 943 LTCC substrate $\left(\varepsilon_{\mathrm{r}}=7.5, \operatorname{Tan} \delta=0.002\right)$. The junction is built in a 12-layer substrate with the thickness of 4.4 mils for each layer. Laminated waveguide is constructed inside the lower eight layers and the microstrip line uses the upper four layers. The cross-section of the laminated waveguide is 140 mils by 32.5 mils. The grid-like sidewall is constructed by via-holes with 3.5 mils in diameter and 15 mils center-to-center distance. The microstrip line used is a 4 mils wide metal strip on the top surface of the substrate with impedance of $100 \Omega$. The detail dimensions of the probe structure are (unit: mils): $\mathrm{R} 0=2.75, \mathrm{R} 1=13, \mathrm{R} 2=4.8, \mathrm{R} 3=5.8, \mathrm{R} 4=7.8$, and $\mathrm{R} 5=7.8$. 


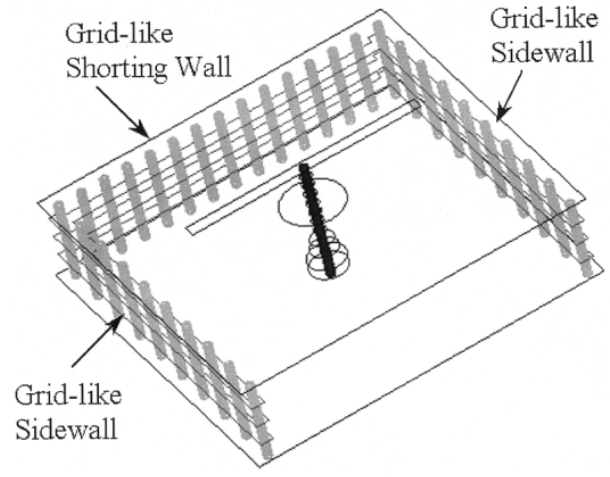

(a)

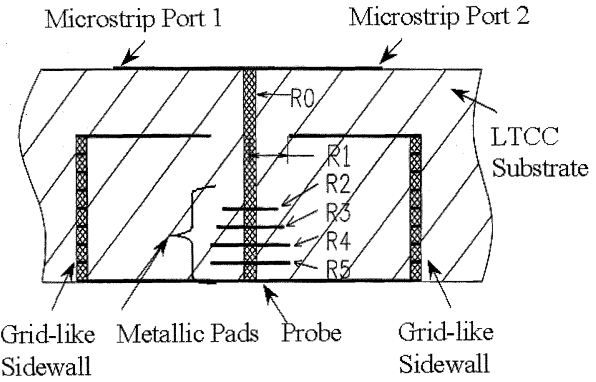

(b)

Fig. 2. Perspective view of the laminated waveguide to microstrip line T-junction: (a) perspective view of the T-junction and (b) cross section of the T-junction.

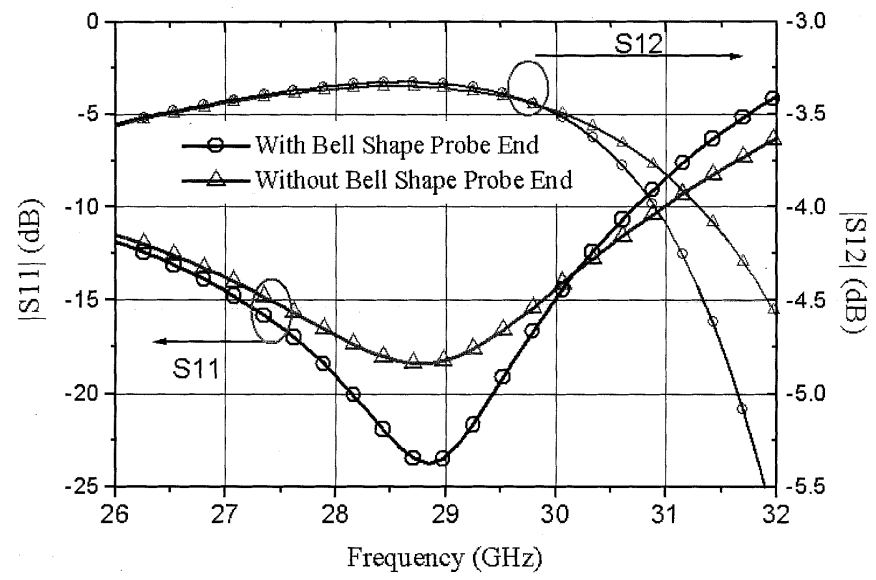

Fig. 3. Simulated result of the T-junction adopting probe with and without bell-shape end.

Fig. 3 shows the simulated result of the proposed T-junction. A T-junction adopting a probe without bell-shape end is also investigated. The distances from center of probe to the shorting wall are 60 mils and 64 mils, respectively, for the probe with and without bell-shape end.

According to the simulated result, a more than $10 \%$ bandwidth defined at $-15 \mathrm{~dB}$ return loss is obtained for the T-junction employing a bell-shape probe end at the center frequency of $29 \mathrm{GHz}$, whereas the insertion loss is about $0.3 \mathrm{~dB}$ at the center frequency

\section{EXPERIMENTAL RESULT}

To measure the performance of the proposed T-junction, a broadband laminated waveguide to WR28 waveguide transition

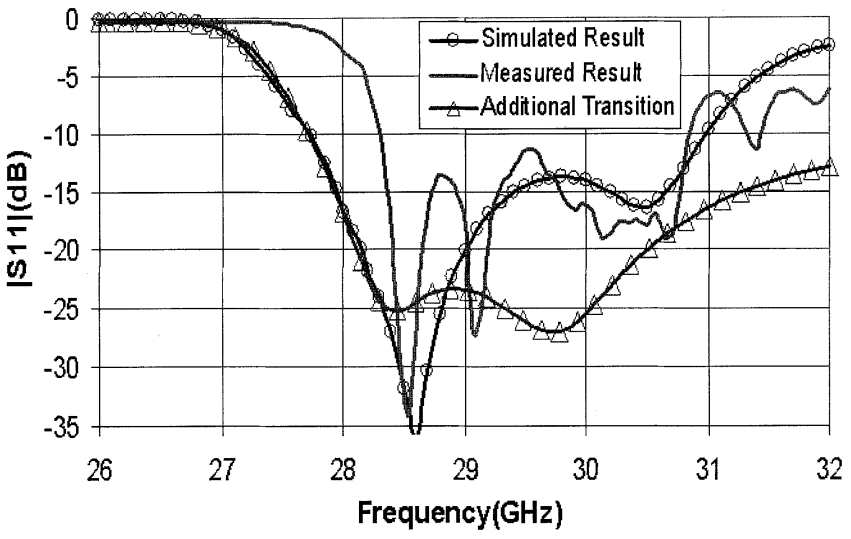

Fig. 4. Simulated and measured results of the cascade module.

is also developed [7], whose simulated reflection coefficient is shown in Fig. 4 and labeled as additional transition. With the additional transition, laminated waveguide of the T-junction is interconnected to a WR-28 air filled waveguide, which can be directly interfaced to HP8510 C vector network analyzer using WR28 calibration kits. Owing to the two completely different type of ports, only the reflection coefficient of the cascaded module can be measured by terminating the microstrip ports with absorbing material. To strictly verify the design, an exactly EM simulation on cascaded module also is carried out. The simulated and measured results of the cascaded module are superposed in Fig. 4. Good agreement can be observed.

\section{CONCLUSION}

An LTCC integrated laminated waveguide to microstrip line $\mathrm{T}$-junction is proposed and verified. Good agreement is obtained between the simulated and the measured refection coefficient. The proposed T-junction is simple and well suitable for being fabricated by LTCC multilayer technology. It can be used in various LTCC integrated modules in mm-wave frequency band. The concept can also be used in the design of a broadband laminated waveguide to microstrip line transition.

\section{REFERENCES}

[1] S. Holzwarth, J. Kassner, R. Kulke, and D. Heberling, "Planar antenna arrays on LTCC-multilayer technology," in Proc. 11th Int. Conf. Antennas and Propagation, vol. 2, 2001, pp. 710-714.

[2] K. Lim, A. Obatoyinbo, M. Davis, J. Laskar, and R. Tummala, "Development of planar antennas in multi-layer packages for RF-system-on-apackage applications," in Dig. Topic Meeting on Electrical Performance of Electronic Packaging, 2001, pp. 101-104.

[3] H. Uchimura, T. Takenoshita, and M. Fujii, "Development of a laminated waveguide," IEEE Trans. Microwave Theory Tech., vol. 46, pp. 2438-2443, Dec. 1998.

[4] D. Deslandes and K. Wu, "Integrated microstrip and rectangular waveguide in planar form," IEEE Microwave Wireless Components Lett., vol. 11, pp. 68-70, Feb. 2001.

[5] H. Uchimura and T. Takenoshita, "A ceramic planar $77 \mathrm{GHz}$ antenna array," in IEEE MTT-S Int. Microwave Symp. Dig., 1999, pp. 453-456.

[6] S. B. Cohn, "Waveguide to coaxial line junction," Proc. IRE, pp. 920-926, Sept. 1947.

[7] Y. Huang and K.-L. Wu, "A broadband LTCC integrated transition of laminated waveguide to air-filled waveguide for millimeter wave application," IEEE Trans. Microwave Theory Tech., vol. 51, pp. 1613-1617, May 2003. 